\title{
Posterior approach to thoracoscopic pulmonary segmentectomy of the dorsal basal segment: A single-institute retrospective review
}

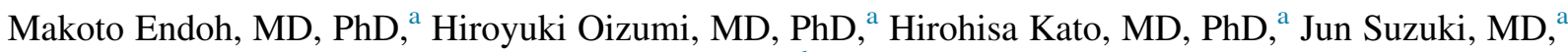 \\ Hikaru Watarai, MD, ${ }^{\mathrm{a}}$ Toshiaki Masaoka, $\mathrm{MD}, \mathrm{PhD},{ }^{\mathrm{b}}$ and Mitsuaki Sadahiro, $\mathrm{MD}, \mathrm{PhD}^{\mathrm{a}}$
}

\section{ABSTRACT}

Objective: Anatomic resection of the dorsal area of the basal segment of the lower lobe is difficult because of the deep location of vessels and bronchi in the parenchyma. This study aimed to describe a novel technique for port-access thoracoscopic segmentectomy of the dorsal (S10) and lateral dorsal segments (S9+10).

Methods: This retrospective study analyzed 20 patients who underwent S10 and S9+10 thoracoscopic segmentectomy via a posterior approach between January 2004 and March 2016. In this approach, the lung parenchyma between S6 and $\mathrm{S} 10$ was divided along V6b,c from the dorsal side of the lower lobe, which exposed the targeted bronchus $(\mathrm{B} 10, \mathrm{~B} 9+10)$ and artery $(\mathrm{A} 10, \mathrm{~A} 9+10)$ and enabled anatomic S10 and S9+10 segmentectomy.

Results: Of the 20 patients, 15 had lung cancer, 3 had metastases, and 2 had benign nodules. The number of segmentectomies of the right $S 10$, right $S 9+10$, left $\mathrm{S} 10$, and left $\mathrm{S} 9+10$ was 5, 5, 1, and 9, respectively. Median operative time was 165 minutes (range, 107-276 minutes). The median duration of chest tube insertion was 1 day (range, 1-2 days). One patient had atelectasis. Median hospital stay was 6 days (range, 3-11 postoperative days). No recurrence or mortality was observed during the median follow-up period of 46 months.

Conclusions: The posterior approach for port-access thoracoscopic segmentectomy at $\mathrm{S} 10$ or $\mathrm{S} 9+10$ is technically challenging, but in our hands it has been feasible. It exposes the targeted bronchus $(\mathrm{B} 10, \mathrm{~B} 9+10)$ and artery (A10, A9+10) and enables anatomic S10 and S9+10 segmentectomy while avoiding inessential parenchymal splitting from the major fissure. ( $\mathrm{J}$ Thorac Cardiovasc Surg 2017;154:1432-9)

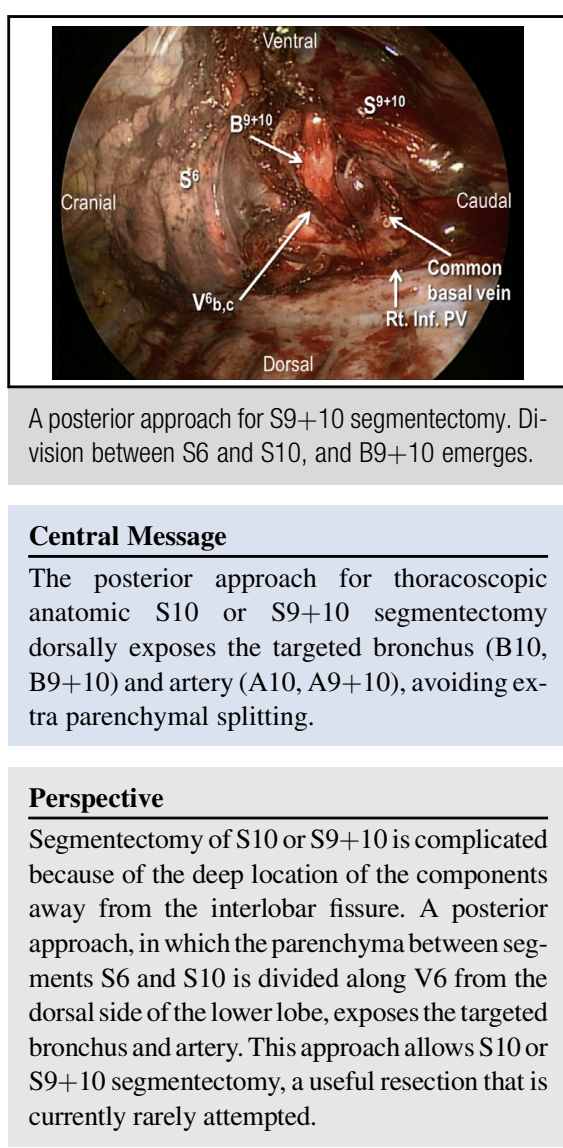

See Editorial Commentary page 1440.
Computed tomography (CT) has enabled the detection of small-sized lung cancers. As a result, the diagnosis of nonsolid tumors with ground-glass opacity, suggesting

From the a Department of Surgery II, Faculty of Medicine, Yamagata University, Yamagata City; and ${ }^{\mathrm{b}}$ Thoracic Division, Tsuruoka Municipal Shonai Hospital, Tsuruoka City, Yamagata Prefecture, Japan.

Read at the 96th Annual Meeting of The American Association for Thoracic Surgery, Baltimore, Maryland, May 14-18, 2016.

Received for publication May 30, 2016; revisions received Feb 9, 2017; accepted for publication March 7, 2017; available ahead of print April 29, 2017.

Address for reprints: Makoto Endoh, MD, PhD, Department of Surgery II, Faculty of Medicine, Yamagata University, 2-2-2 Iida-Nishi, Yamagata City, Yamagata Prefecture 990-9585, Japan (E-mail: m-endoh@med.id.yamagata-u.ac.jp).

$0022-5223 / \$ 36.00$

Copyright (c) 2017 by The American Association for Thoracic Surgery

http://dx.doi.org/10.1016/j.jtcvs.2017.03.120 noninvasive lung cancer with good prognosis, is increasing. ${ }^{1-4}$ Therefore, limited resection as anatomic segmentectomy is increasingly considered for resection of such lesions. Although it is reasonable to perform lessinvasive resection of a smaller volume of lung tissue thoracoscopically, this type of surgery often involves complicated procedures. Consequently, published reports

- Scanning this QR code will take you to supplemental videos for the article.

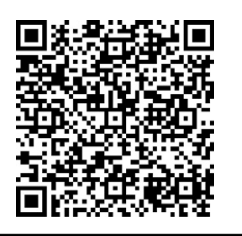




\section{Abbreviations and Acronyms \\ 3-D = 3-dimensional \\ $\mathrm{CT}=$ computed tomography \\ MDCT = multidetector computed tomography \\ SAMURAI $=$ Segmentectomy Achieved by MDCT for Use in Respective Anatomical Interpretation}

on segmentectomy under total thoracoscopic visualization are limited. ${ }^{5-9}$ Anatomic thoracoscopic segmentectomy requires accurate delineation of the inflation-deflation line and identification of intersegmental veins, which are reliable landmarks of the intersegmental plane. ${ }^{10-12}$

We previously reported the importance of 3-dimensional (3-D) simulation using multidetector computed tomography (MDCT) for interpretation of individual segmental anatomy: the Segmentectomy Achieved by MDCT for Use in Respective Anatomical Interpretation (SAMURAI) technique. ${ }^{12}$ After we introduced SAMURAI using intraoperative simulated navigation, the number of segmentectomies classified as difficult increased, including dorsal segment (S10) and lateral dorsal segment (S9+10) segmentectomies. Conventionally, the surgical approach for these segments has been designed from the interlobar fissure, dividing the lung parenchyma between the superior segment (S6) and the ventral segment (S8) to expose the deeply located targeted pulmonary artery (A10 or $\mathrm{A} 9+10)$ and bronchus (B10 or $\mathrm{B} 9+10) \cdot{ }^{13,14}$ We have developed a new posterior approach for thoracoscopic segmentectomy of the same segment after analyzing 3-D CT angiography and bronchography. ${ }^{15}$

The objective of this study was to report our experience with this new approach for port-access thoracoscopic anatomic segmentectomy of the dorsal basal segment or the lateral dorsal basal segment and to determine the feasibility of this approach on the basis of the initial results of 20 resections.

\section{PATIENTS AND METHODS \\ Patients}

All of the surgeries were performed by 1 general thoracic surgeon (H.O.) with assistant surgeons. Among 256 patients undergoing portaccess thoracoscopic anatomic segmentectomy between January 2004 and March 2016, 20 who underwent thoracoscopic S10 or S9+10 segmentectomy through the posterior approach were included to evaluate the utility of the posterior approach for pulmonary segmentectomy of the basal segment. Patients' data were retrospectively analyzed. The present study was approved, the need for written informed consent from each patient was waived by the Institutional Review Board, and the study was conducted in accordance to the concepts of the Declaration of Helsinki.

\section{Selection Criteria}

We considered specific patients for this technique: patients with a T1 N0 small peripheral lung cancer, ${ }^{16}$ a lung metastasis, a ground-glass opacity, or an indeterminate lung lesion that might be benign. We also consider the patients who are "compromised" in that they are considered to be poor candidates for lobectomy because of limited cardiopulmonary reserve or other comorbidities. Wedge resection was considered inappropriate in all cases because of the tumor size or location in the deep parenchyma. Resected specimens were examined histopathologically, and histologic typing was done according to the World Health Organization classification. ${ }^{17}$ In cases of malignancy, we designed the resection lines with a larger surgical margin than the tumor diameter (at least $2 \mathrm{~cm}$ ). In case of insufficient surgical margin of the resected specimen, we performed an additional wedge resection using stapler. Surgical-pathologic staging was according to the TNM Classification of Malignant Tumors, Seventh Edition, published by the International Union Against Cancer and the American Joint Committee on Cancer. ${ }^{18}$ If the cancer was in S10 near the S9 border, we selected the S9+S10 bisegmentectomy (Figure 1, A).

\section{Three-Dimensional Computed Tomography Imaging and Simulated Navigation Method}

The 3-D images were processed using preoperatively acquired volume data derived from CT. We also used the free software or work station. Processing required no more than 7 minutes. ${ }^{12}$ The 3-D images were created and manipulated during surgery with the free software or work station of the CT equipment. Thus, we performed the operations while comparing and contrasting the simulation under real-time conditions. ${ }^{11,12,19,20} \mathrm{We}$ think that grasping the anatomic relationship between the tumor and the neighboring structures is important in the CT findings and 3-D CT imaging. The 3-D CT simulation images were used to identify the (1) segmental arterial branches, (2) intersegmental veins, and (3) venous branches in the affected segment.

\section{Operative Procedure}

Under the guidance of the previously mentioned simulated navigation, we applied the SAMURAI technique. ${ }^{12}$ We collapsed the lung on the operating side and anesthetized the patient under differential ventilation. During the procedure, the surgeon stood on the dorsal side of the patient and the assistants stood on the ventral side. The image, on the assistants' side, was rotated $180^{\circ}$. Four ports ( 1 with a diameter of 20 or $15 \mathrm{~mm}$ [flexible] and 3 with a diameter of $5 \mathrm{~mm}$ ) were prepared for the port access technique.

We first exposed the inferior pulmonary vein and its branches, the superior (V6) and the common basal vein, with traction of the lower lobe anteriorly (Figure $1, B$ ). The intersegmental plane between S6 and S10 was divided using electrocautery or an energy device along V6b and V6c, which were the intersegmental veins between the superior (S6) and basal segments. An ultrasonically activated device or bipolar tissue sealing and dividing system were primarily used to divide the smaller segmental vessel branches after proximal ligation of the vessels. Thereafter, the targeted bronchus (B10 or B9+10) was exposed and the targeted pulmonary artery (A10 or $\mathrm{A} 9+10$ ) was exposed posteriorly (Figures $1, B$, and $2, B$ ). We secured the targeted bronchus after parenchymal dissection of approximately $3 \mathrm{~cm}$ along V6b and V6c. The targeted bronchus was secured using the bronchoscope and was looped with a suture. The targeted bronchus was then divided using the resected segment inflation method, ${ }^{21,22}$ in which the affected segment was inflated and the reserved lung was deflated using the slip-knot bronchial ligation method ${ }^{23}$ or the high-frequency jet ventilation via the bronchoscope technique. ${ }^{10}$ The slip-knot technique was used for bronchial ligation and for visualization of the anatomic plane (Figure 2, Video 1). After looping the bronchus with monofilament suture, a slip knot was made outside the thorax. During bilateral lung ventilation, 1 end of the string was pulled, and the knot slipped to reach the bronchus without a knot-pusher. Bronchial ligation was then performed to block the outflow of segmental air while the segment remained inflated, whereas the other segments collapsed. The difference between the inflated and deflated lung parenchyma formed the demarcation line. The targeted artery 

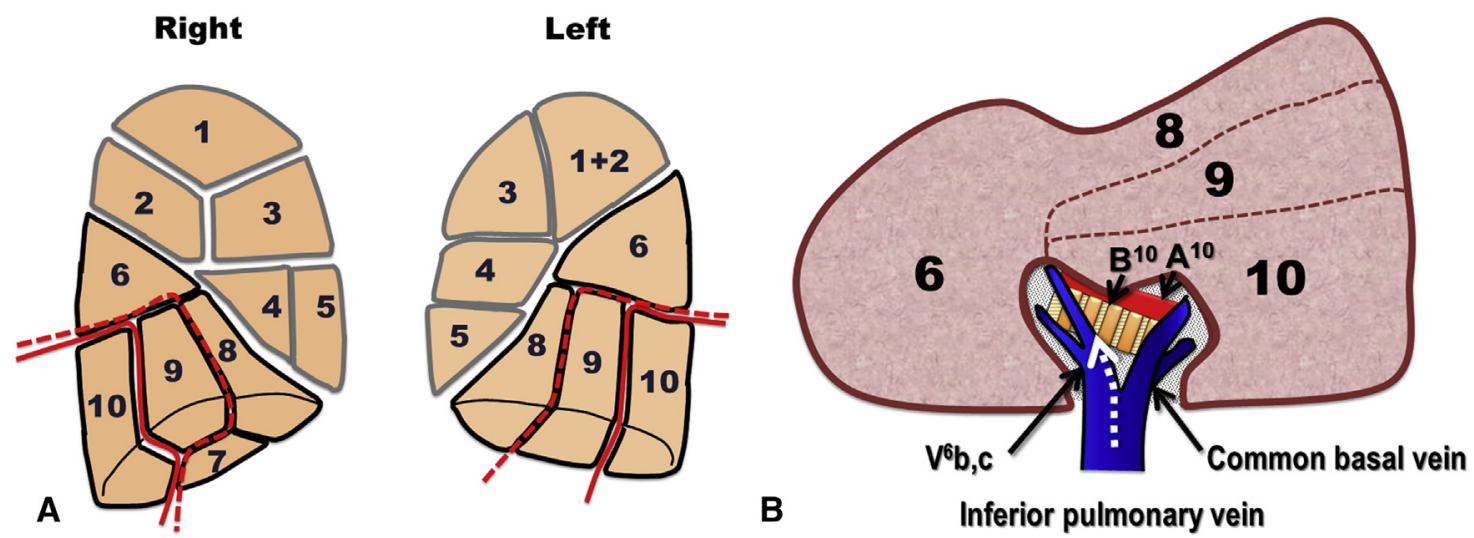

FIGURE 1. Diagram of the pulmonary segments. A, The continuous line indicates the dorsal basal segmentectomy (S10), and the broken line divides the lateral dorsal basal segment (S9+10). The superior segment (S6) connects to the ventral basal segment (S8). B, Schema of a posterior approach for S10 or S9+10 segmentectomy. The inferior pulmonary vein consists of the superior (V6) and common basal vein, which branches to superior and inferior basal veins. Dividing the lung parenchyma along V6b,c posteriorly exposed the targeted bronchus (B10, B9+10), and the targeted artery (A10, A9+10) was exposed behind it.

was divided before or after division of the bronchus. The posterior vein (V10) and intrasegmental veins, which entered the resected segment, were divided and resected after identification. The demarcation line between the inflated and deflated lung parenchyma became visible; thereafter, the surgeon dissected the parenchyma along the inflation-deflation line or the intersegmental veins, using electrocautery or tissue sealer. The same device was used to incise the lung parenchyma along the intersegmental vein around the hilum; then, after releasing approximately one third of the hilar parenchyma, we used staplers of thick range cartridge, depending on the thickness of the parenchyma. In some cases, we incised the peripheral lung using electrocautery along the inflation-deflation line and applied the stapler to divide the residual parenchyma. Postoperative CT confirmed

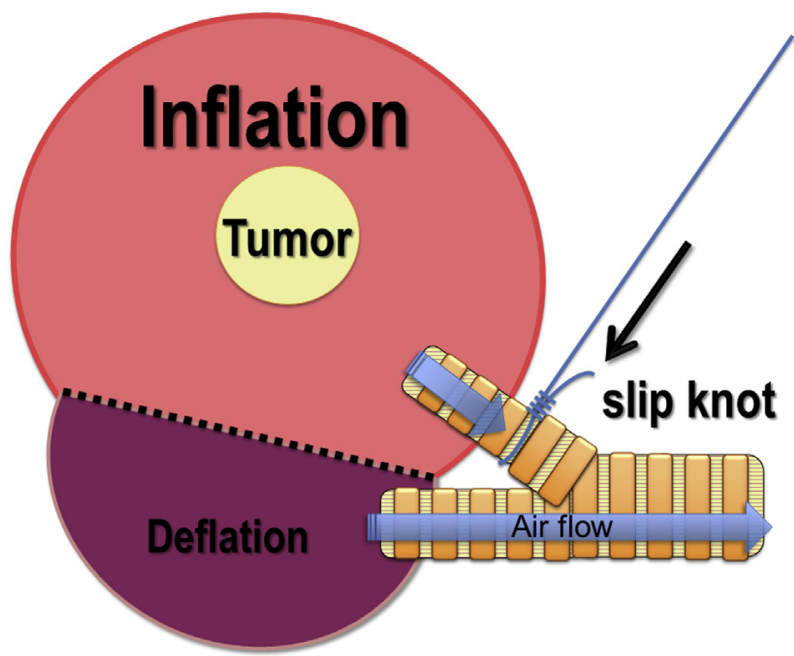

FIGURE 2. Slip-knot bronchial ligation for creating inflation-deflation line. Both lungs were ventilated, and the slip knot made outside thorax was fully pulled to ligate the bronchus with full expansion of the lungs. The affected segment remained inflated while the other segments appeared collapsed. The difference between the inflated and deflated lung parenchyma formed the demarcation line. The surgeon then dissected the parenchyma along the inflation-deflation line. The dotted line indicates the inflation-deflation line. the adequacy of resection in all patients. Dorsal lateral segment resection of the right lower lobe, a representative case of the posterior approach, is presented in Figures 3 and 4 and Video 2.

\section{Statistical Analysis}

Statistical analysis was performed using Microsoft Excel software (Microsoft, Redmond, Wash). Quantitative variables were reported as mean \pm standard deviation or median and range.

\section{RESULTS}

Patients' characteristics are listed in Table 1. Histologically, the majority of patients had a diagnosis of malignancy

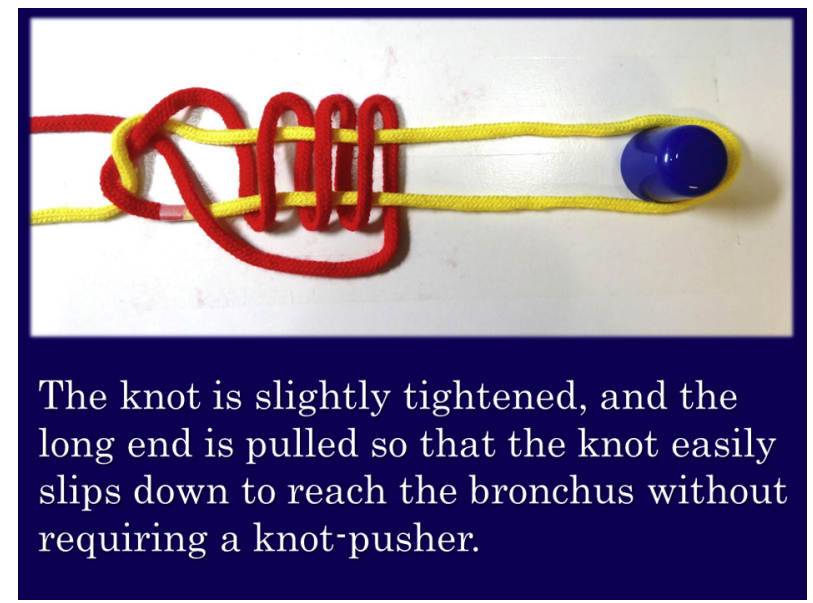

VIDEO 1. The slip knot is a modified Roeder knot customized for bronchial ligation. To make the knot, create a half hitch and hook this throw to the bronchus using the third finger of the left hand. Wrap the end 3 times around the limbs of the loop. Bring the end back, hold this end between the thumb and third finger, and then pass through the loop that was made with the hitch. The knot is slightly tightened, and the long end of the monofilament suture is pulled so that the knot easily slips down to reach the operative field without requiring a knot-pusher. Video available at: http://www.jtcvsonline.org/article/S0022-5223(17)30641-4/addons. 

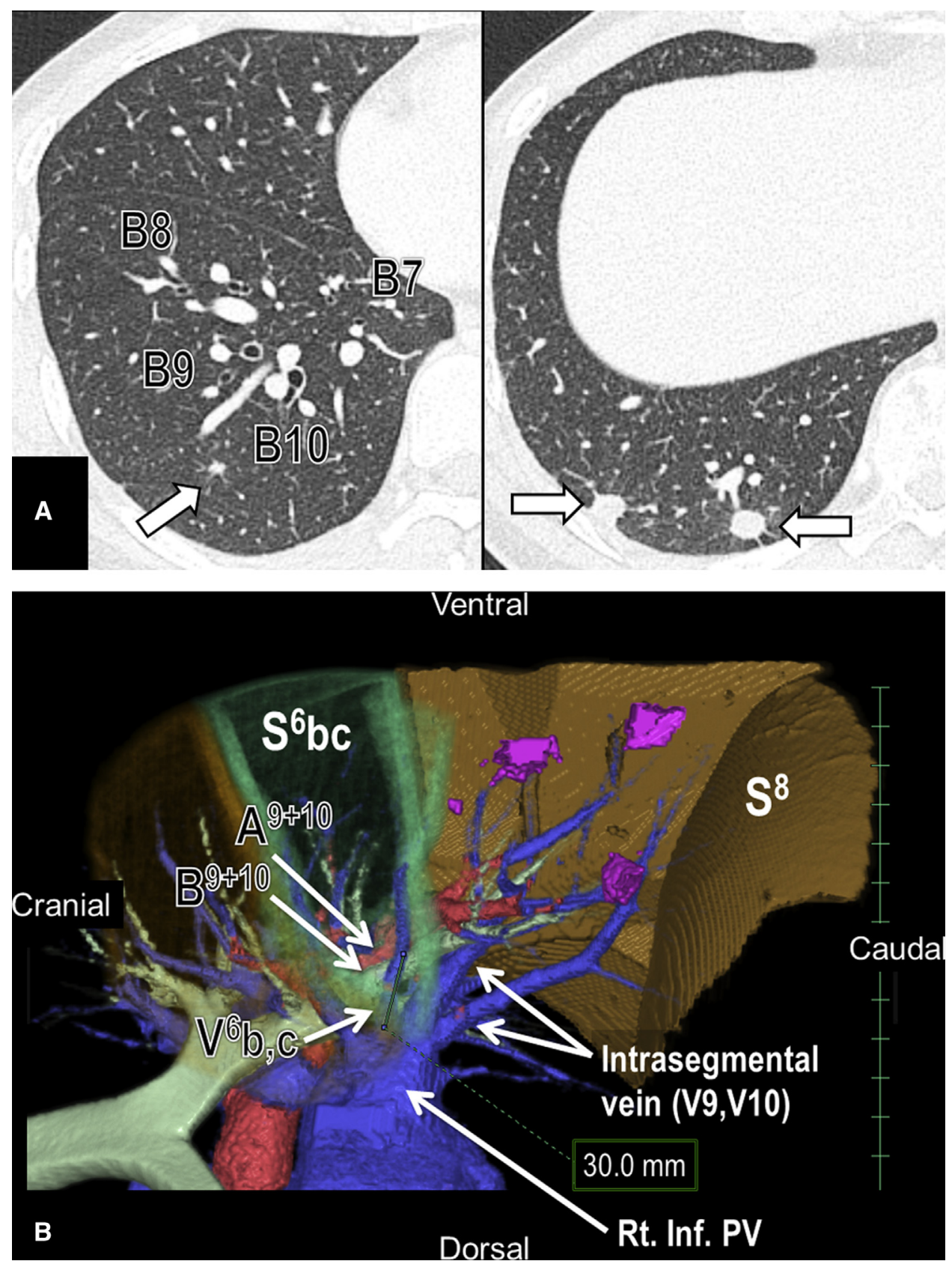

FIGURE 3. Dorsal lateral segment (S9+10) resection of the right lower lobe. A representative case of the posterior approach. A, CT image showing 1 nodule measuring $0.5 \mathrm{~cm}$ and 3 solid nodules measuring $1 \mathrm{~cm}$ each, located in S9 and S10. Arrow indicates nodules (metastases). B, 3-D CT angiography and bronchial tree with a marking of the tumors. The calculated distance from the start of $\mathrm{V} 6 \mathrm{~b}, \mathrm{c}$ to the targeted bronchus (B9+10) was $3 \mathrm{~cm}$. $P V$, Pulmonary vein.

$(18 / 20,90 \%)$, with 15 patients diagnosed with lung cancer after introduction of the posterior approach. In this group, pathologic examination showed that curative resections were achieved with free surgical margins in all patients. Median tumor size was $1.4 \mathrm{~cm}$ (range, $1-2.5 \mathrm{~cm}$ ) in patients with lung cancer and $2.6 \mathrm{~cm}$ (range, 2.3-3.5 cm) in patients with pulmonary metastasis. The median surgical margin distance was $2 \mathrm{~cm}$ (range, $0.5-3 \mathrm{~cm}$ ) and $3.5 \mathrm{~cm}$ (no range), respectively. Of the 15 cases with lung cancer, hilar lymphadenectomy was performed in 8 cases, hilar and mediastinal lymphadenectomy were performed in 6 cases, and hilar lymph node sampling was performed in 1 case. Median dissected lymph node count was 6 (range, 1-33). No lymph node metastases were detected in any patient. The area of lymphadenectomy differed between patients because of the difference in their clinical staging and background.

In regard to the location of affected segments, right- and left-side segmentectomies were performed in an equal number of patients. A total of 14 patients underwent lateral 

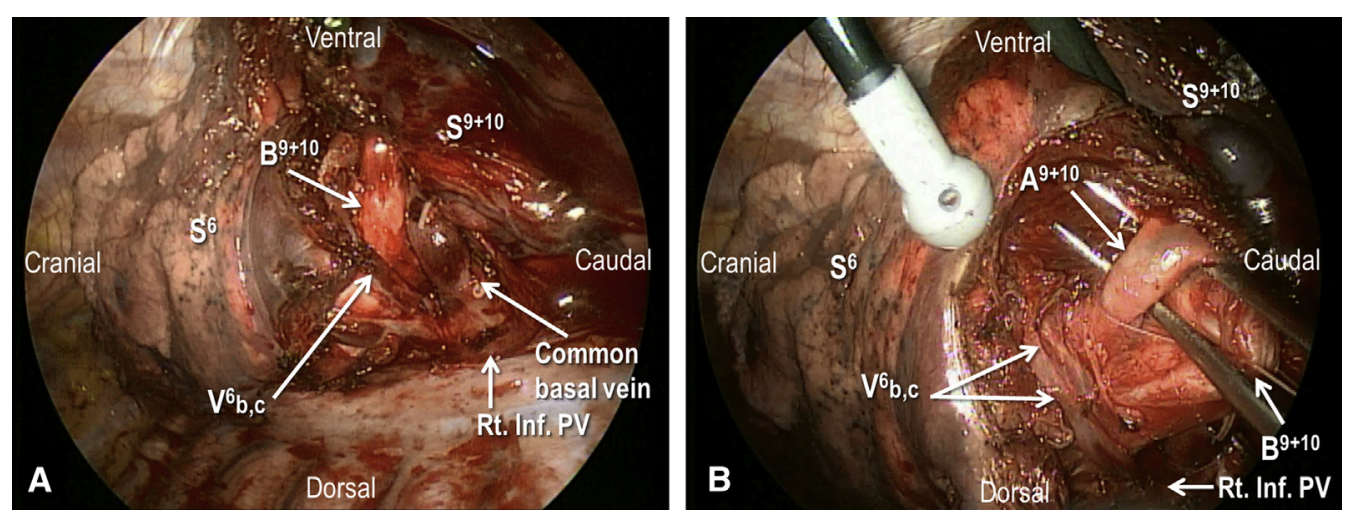

FIGURE 4. Operative view of the patient. A, Intersegmental plane (S6-S10) was divided along the intersegmental vein (V6b,c) from the right inferior pulmonary vein. B, Targeted bronchus $(\mathrm{B} 9+10)$ was exposed, and the targeted pulmonary artery $(\mathrm{A} 9+10)$ was exposed behind it. $P V$, Pulmonary vein.

dorsal segmentectomy (S9+10), and 6 patients underwent dorsal segmentectomy (S10).

Operative results are shown in Table 2. Median operative times before and after introduction of the posterior approach were 259 minutes (range, 22-425 minutes) and 158 minutes (range, 107-276 minutes), respectively. Median intraoperative blood loss was $175 \mathrm{~g}$ (range, 132-200 g) and $74 \mathrm{~g}$ (range, 30-324 g), respectively. Median duration of chest tube insertion was 1 day (range, 1-2 days). One patient had postoperative atelectasis due to productive sputum in the airway from chronic bronchitis. Two of the 20 cases had oncological conversion. Conversion to lobectomy was performed in 1 case with preoperatively suspected metastasis. The tumor in the segmentectomy specimen was revealed to be a primary lung cancer by frozen section. Another patient required additional bronchial wedge resection, and bronchoplasty because remnant carcinoma in situ was observed in the resected specimen. The slip-knot technique $^{23}$ was applied to 12 of 20 patients, and all of them had good visualization of the inflation-deflation line, which enabled intersegmental division using electrocautery or staples along the demarcation line. The conversion-associated surgical technique was not performed in this series, and all lesions were included in the resected segment; therefore, the success rate of the posterior approach for thoracoscopic segmentectomy of the dorsal basal segment was $100 \%$. Median hospital stay was 6 days (range, 3-11 days). No recurrence or mortality was observed during the median follow-up period of 46 months (range, 15-89 months).

\section{DISCUSSION}

The number of reports on small-sized, minimally invasive lung cancer has recently grown. It is increasingly being recognized that wedge resection of these tumors results in a good prognosis, especially in the case of nonsolid tumors. ${ }^{1-4}$ In the case of noninvasive lung cancers located deep within the parenchyma, thoracoscopic wedge resection with sufficient preservation of the margin is impossible. Segmentectomy can be considered in such cases, especially in compromised patients who would do poorly with lobectomy. We consider that pulmonary function can be preserved to a greater degree by combining segmentectomy with less-invasive thoracoscopic surgery. Most reports on thoracoscopic pulmonary segmentectomy usually are limited to anatomically easily accessible segments, such as lingular segments and lower lobe superior segments. ${ }^{5,6,8,9,24,25}$ Intersegmental dissection in such segmentectomies is not procedurally difficult because dissection can be performed using stapling devices along the intersegmental plane. However, this procedure is difficult to apply to other segments. For most intersegmental surfaces, thoracotomy or mini-thoracotomy is necessary. Both involve dissection that relies on tactile sense or direct vision. Formerly, video-assisted thoracoscopic lobectomies were performed in some cases of ground-glass opacity, if requested by patients. Factors influencing the outcome of thoracoscopic pulmonary segmentectomy include intersegmental determination and difficulty in dissection. Visualization of the inflationdeflation line is reportedly useful for determination of the intersegmental plane and dissection, ${ }^{21,22}$ and this procedure is now applied for less-invasive surgery; however, mini-thoracotomy is required for direct visualization. ${ }^{10}$ The presence of an inflation-deflation line that approximates a parenchymal demarcation line was demonstrated in $86 \%$ of 89 patients after introduction of the slip-knot technique. ${ }^{23}$ Visualization of the inflation-deflation line also enabled us to perform precise subsegmentectomy. ${ }^{20}$ In the current study, 12 of 20 patients had good visualization of the inflation-deflation line, which enabled intersegmental division using electrocautery or staples along the demarcation line. In addition, the inflation-deflation line in patients with poor pulmonary conditions, such as in patients with chronic pulmonary emphysema or wet airways retaining mucus, is not easily visualized.

$\mathrm{CT}$ is rapidly being replaced by MDCT to facilitate easier diagnosis and visualization of the anatomy based on 3-D 


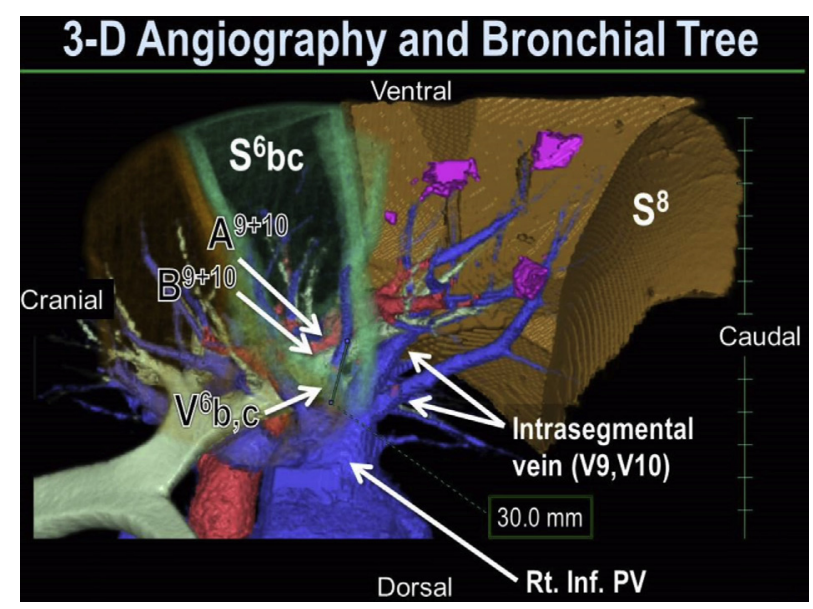

VIDEO 2. A posterior approach for the dorsal lateral segment $(\mathrm{S} 9+10)$ resection of the right lower lobe. A 33-year-old man had 5 metastatic lesions derived from a testicular tumor in the $\mathrm{S} 9+10$ as shown in Figure 2, $A$. Two flexible $11.5-\mathrm{mm}$, one $5-\mathrm{mm}$, and one $20-\mathrm{mm}$ soft ports were inserted. We first exposed the inferior pulmonary vein and its branches, the superior (V6) and the common basal vein, after turning the lower lobe anteriorly. The intersegmental plane (S6-S10) was divided along the intersegmental vein $(\mathrm{V} 6 \mathrm{~b}, \mathrm{~V} 6 \mathrm{c})$ from the right inferior pulmonary vein. The key to this procedure is to release the vascular sheath and dissect the parenchyma using a sealing device. Thereafter, the targeted bronchus $(\mathrm{B} 9+10)$ was exposed and the targeted pulmonary artery $(\mathrm{A} 9+10)$ was exposed behind it. We encircled the bronchus (B9+10) using monofilament polypropylene thread. A slip-knot was made outside the thorax, and whole lung ventilation was performed. During lung ventilation, 1 end of the string was pulled, and the knot slipped to reach the bronchus without a knot-pusher. The outflow of segmental air was blocked and the $\mathrm{S} 9+10$ remained expanded, whereas the other segments collapsed. The bronchus was then divided using a stapler. The inflation-deflation lines became gradually distinct. Then, we further dissected the pulmonary parenchyma along the intersegmental vein or the inflationdeflation line. The intersegmental veins were preserved, and intrasegmental veins were divided. The peripheral lung was dissected with a stapler. The operative time was 157 minutes. Air leakage was not observed, and the chest tube was removed on postoperative day 1 . Video available at: http://www. jtcvsonline.org/article/S0022-5223(17)30641-4/addons.

imaging. ${ }^{19,26-28}$ SAMURAI is a segmentectomy technique in which the location of the tumor resection margin is estimated on the basis of CT images, and the targeted blood vessels are determined in 3-D. ${ }^{7,12}$ This imaging procedure does not cost more because contrast mediumenhanced CT is performed routinely to evaluate lymph node enlargement in patients with lung cancer in our institution. By using this procedure to identify the intersegmental veins and then dissecting along them, it became possible to perform segmentectomy via a completely thoracoscopic approach, even for the anatomically difficult segments.

In dorsal basal segmentectomy (S10) or lateral dorsal basal segmentectomy (S9+10) from the interlobar fissure approach anteriorly, dividing the lung parenchyma between the superior segment (S6) and the ventral segment (S8) is a
TABLE 1. Patients' characteristics

\begin{tabular}{lc}
\hline \multicolumn{1}{c}{ Characteristic } & Data $(\mathbf{n}=\mathbf{2 0})$ \\
\hline Age $(\mathrm{y})$ & \\
Range & $15-81$ \\
Median & 66 \\
Male/female & $9 / 11$ \\
Clinical diagnosis & \\
Lung cancer & 15 \\
Invasive adenocarcinoma & 3 \\
Minimally invasive & 2 \\
$\quad$ adenocarcinoma & \\
Adenocarcinoma in situ & 6 \\
Squamous cell carcinoma & 3 \\
Other & 1 \\
Metastasis & 3 \\
Benign & 2 \\
Bronchial atresia & 1 \\
Pulmonary sequestration & 1 \\
\hline
\end{tabular}

conventional maneuver to expose the deep targeted pulmonary artery (A10 or A9+10) and the targeted bronchus (B10 or B9+10) located posteriorly. ${ }^{13,14}$ These divided areas were originally connected to each other, and preservation without division, if possible, seems to be less invasive. Inessential parenchymal splitting from the major fissure might result in the development of air leakage or torsion of the remnant segments.

The dorsal basal artery in particular is located deeply in the lower lobe because it is a dorsal branch of the lateral dorsal basal artery. Therefore, pursuing the delicate, deeply located pulmonary artery with resection of lung parenchyma from the origin of the lobar pulmonary artery to the peripheral segmental artery is technically complicated.

TABLE 2. Surgical results

\begin{tabular}{lc}
\hline Variables & After $(\mathbf{n}=\mathbf{2 0})$ \\
\hline Operative time (min) & $107-276$ \\
$\quad$ Range & 165 \\
Median & \\
Bleeding (g) & $30-324$ \\
$\quad$ Range & 74 \\
$\quad$ Median & \\
Chest tube duration (d) & $1-2$ \\
$\quad$ Range & 1 \\
$\quad$ Median & \\
Hospital stay (d) & $3-11$ \\
$\quad$ Range & 6 \\
$\quad$ Median & \\
Complication (n) & 1 \\
$\quad$ Atelectasis & 0 \\
Morbidity (n) & 0 \\
\hline Mortality (n) & \\
\hline
\end{tabular}


On the contrary, division between the superior segment and the basal segment is easy, because hyperlobulation in the lower lobe is observed on occasion. ${ }^{13}$ The landmark used for its division is the intersegmental veins (V6b and V6c) branching from the superior pulmonary vein. We developed a posterior approach for thoracoscopic dorsal basal segmentectomy and lateral dorsal basal segmentectomy through analyzing 3-D CT angiography with or without bronchography. By dividing the intersegmental plane between S6 and S10 along V6b and V6c, which are the intersegmental veins between the superior segment and the basal segment from the inferior pulmonary vein peripherally, the targeted bronchus (B10 or B9+10) is exposed first and can be recognized as having a less-flexible and funicular structure. The key to this procedure is to release the vascular sheath and dissect the parenchyma using a sealing device. The segmental bronchus usually tolerates blunt dissection without damage, and thus we can safely reach the pulmonary hilum. Because the targeted segmental artery (A10 or A9+10) is located behind the bronchus and is exposed after dissection of the bronchus, the technique is useful in preventing arterial injury. The targeted bronchus was divided after the resected segment inflation method ${ }^{21,22}$ in which the affected segment was inflated and the reserved lung was deflated using the slip-knot bronchial ligation method that we introduced in $2010 .^{23}$ The demarcation line between the inflated and deflated lung parenchyma became visible; thereafter, the surgeon dissected the parenchyma along the inflationdeflation line or the intersegmental vein.

All procedures were performed thoracoscopically without the conversion to open thoracotomy using this posterior approach. No mortality associated with this approach was observed. Although atelectasis developed postoperatively in 1 patient because of retained sputum, she recovered without any intervention. Therefore, this approach was feasible for the application of port-access thoracoscopic segmentectomy of the dorsal basal segment. The merit of this procedure is that inessential parenchymal splitting from the major fissure is avoided. However, this approach might be insufficient for lymph node dissection at the hilum. Thus, we limited the indication of this technique for early-stage lung cancer and pulmonary metastasis not requiring lymph node dissection or if dissection was needed, we manipulated the lymph nodes from the bilateral intersegmental plane and interlobar side. We applied this procedure in a limited fashion for ground-glass opacity nodules in patients with lung cancer; therefore, we believe it is reasonable to omit further nodal dissection, because such lung tumors are usually diagnosed as adenocarcinoma in situ or minimally invasive adenocarcinoma. Mediastinal lymph node dissection was performed if the tumor included a solid component comprising more than $30 \%$ of the tumor.

In the present study, the maximum operative time was 276 minutes for 1 case (right dorsal basal segment, the second case after introduction of posterior approach). This case had biases, such as difficulty in performing the technique and the learning curve. The hospital stay in this series might seem to be long; however, in Japan, postoperative hospital stay is longer than in European or North American countries, because the medical health insurance system is less expensive. Therefore, postoperative hospital stay is longer than it could be if we were more firm in our resolve to discharge patients when the chest tube was removed.

\section{Study Limitations}

One study limitation is its retrospective nature, being conducted in a single institute with a single surgeon performing all the operations. Interpretation of these results may not necessarily be generalizable to the more complex clinical settings, and prospective studies are necessary to delineate the merits of this posterior approach for dorsal basal segmentectomy. There are some biases, such as the learning curve in the use of newly developed instruments.

\section{CONCLUSIONS}

A posterior approach for port-access thoracoscopic pulmonary segmentectomy for the basal segment enabled us to accomplish anatomic segmentectomy for the dorsal and the lateral dorsal segment, which have been considered to be complicated and difficult to resect, while avoiding inessential parenchymal splitting from the major fissure. 3-D CT imaging and the slip-knot ligation technique aid in performing this operation more easily. The indication of this technique might be limited to early-stage lung cancer and pulmonary metastasis not requiring lymph node dissection because of insufficiency for hilar lymph node dissection.

\section{Conflict of Interest Statement}

Authors have nothing to disclose with regard to commercial support.

\section{References}

1. Okada M, Nishio W, Sakamoto T, Uchino K, Hanioka K, Ohbayashi C, et al. Correlation between computed tomographic findings, bronchioloalveolar carcinoma component, and biologic behavior of small-sized lung adenocarcinomas. $J$ Thorac Cardiovasc Surg. 2004;127:857-61.

2. Yoshida J, Nagai K, Yokose T, Nishimura M, Kakinuma R, Ohmatsu H, et al. Limited resection trial for pulmonary ground-glass opacity nodules: fifty-case experience. J Thorac Cardiovasc Surg. 2005;129:991-6.

3. Nakata M, Sawada S, Yamashita M, Saeki H, Kurita A, Takashima S, et al. Objective radiologic analysis of ground-glass opacity aimed at curative limited resection for small peripheral non-small cell lung cancer. J Thorac Cardiovasc Surg. 2005; 129:1226-31.

4. Mun M, Kohno T. Efficacy of thoracoscopic resection for multifocal bronchioloalveolar carcinoma showing pure ground-glass opacities of $20 \mathrm{~mm}$ or less in diameter. J Thorac Cardiovasc Surg. 2007;134:877-82.

5. Shiraishi T, Shirakusa T, Iwasaki A, Hiratsuka M, Yamamoto S, Kawahara K Video-assisted thoracoscopic surgery (VATS) segmentectomy for small peripheral lung cancer tumors: intermediate results. Surg Endosc. 2004;18:1657-62.

6. Atkins BZ, Harpole DH Jr, Mangum JH, Toloza EM, D’Amico TA, Burfeind WR Jr. Pulmonary segmentectomy by thoracotomy or thoracoscopy: reduced hospital length of stay with a minimally-invasive approach. Ann Thorac Surg. 2007;84:1107-12. 
7. Oizumi H, Kanauchi N, Kato H, Endoh M, Takeda S, Suzuki J, et al. Total thoracoscopic pulmonary segmentectomy. Eur J Cardiothorac Surg. 2009;36:374-7.

8. Ghaly G, Kamel M, Nasar A, Paul S, Lee PC, Port JL, et al. Video-assisted thoracoscopic surgery is a safe and effective alternative to thoracotomy for anatomic segmentectomy in patients with clinical stage I non-small cell lung cancer. Ann Thorac Surg. 2016;101:465-72.

9. Kodama K, Higashiyama M, Okami J, Tokunaga T, Imamura F, Nakayama T, et al. Oncologic outcomes of segmentectomy versus lobectomy for clinical T1a N0 M0 non-small cell lung cancer. Ann Thorac Surg. 2016;101:504-11.

10. Okada M, Mimura T, Ikegaki J, Katoh H, Itoh H, Tsubota N. A novel videoassisted anatomic segmentectomy technique: selective segmental inflation via bronchofiberoptic jet followed by cautery cutting. J Thorac Cardiovasc Surg. 2007:133:753-8.

11. Oizumi H, Endoh M, Takeda S, Suzuki J, Fukaya K, Sadahiro M. Anatomic lung segmentectomy simulated by computed tomographic angiography. Ann Thorac Surg. 2010;90:1382-3.

12. Oizumi H, Kanauchi N, Kato H, Endoh M, Suzuki J, Fukaya K, et al. Anatomic thoracoscopic pulmonary segmentectomy under 3-dimensional multidetector computed tomography simulation: a report of 52 consecutive cases. J Thorac Cardiovasc Surg. 2011;141:678-82.

13. Arai T, Shiozawa M. Pulmonary Resections. Tokyo: Asakura Shoten-Verlag; 1992.

14. Nomori H, Okada M. Illustrated Textbook of Anatomic Pulmonary Segmentectomy. Berlin: Springer-Verlag; 2012.

15. Oizumi H, Kato H, Fukaya K, Shiota H, Sadahiro M. A posterior approach for lateral posterior basal bisegmentectomy of the lower lobes. J Jpn Assoc Chest Surg. 2011;25:235-7 (in Japanese).

16. Kodama K, Doi O, Higashiyama M, Yokouchi H. Intentional limited resection for selected patients with T1 N0 M0 non-small-cell lung cancer: a single institution study. J Thorac Cardiovasc Surg. 1997;114:347-53.

17. Travis WD, Brambilla E, Muller-Hermelink HK, Harris CC. Pathology and Genetics of tumours of the Lung, Pleura, Thymus and Heart. Lyon, France: IARC Press; 2004.

18. Travis WD, Brambilla E, Noguchi M, Nicholson AG, Geisinger KR, Yatabe Y, et al. International Association for the Study of Lung Cancer/American Thoracic Society/European Respiratory Society: international multidisciplinary classification of lung adenocarcinoma. J Thorac Oncol. 2011;6:244-85.
19. Chan EG, Landreneau JR, Schuchert MJ, Odell DD, Gu S, Pu J, et al. Preoperative (3-dimensional) computed tomography lung reconstruction before anatomic segmentectomy or lobectomy for stage I non-small cell lung cancer. J Thorac Cardiovasc Surg. 2015;150:523-8.

20. Kato H, Oizumi H, Inoue T, Oba E, Nakamura K, Hayashi J, et al. Port-access thoracoscopic anatomical lung subsegmentectomy. Interact Cardiovasc Thorac Surg. 2013;16:824-9.

21. Tsubota N, Ayabe K, Doi O, Mori T, Namikawa S, Taki T, et al. Ongoing prospective study of segmentectomy for small lung tumors. Ann Thorac Surg. 1998;66: 1787-90.

22. Tsubota N. An improved method for distinguishing the intersegmental plane of the lung. Surg Today. 2000;30:963-4.

23. Oizumi H, Kato H, Endoh M, Inoue T, Watarai H, Sadahiro M. Slip knot bronchial ligation method for thoracoscopic lung segmentectomy. Ann Thorac Surg. 2014;97:1456-8.

24. D'Amico TA. Thoracoscopic segmentectomy: technical considerations and out comes. Ann Thorac Surg. 2008;85:S716-8.

25. Shapiro M, Weiser TS, Wisnivesky JP, Chin C, Arustamyan M, Swanson SJ Thoracoscopic segmentectomy compares favorably with thoracoscopic lobectomy for patients with small stage I lung cancer. J Thorac Cardiovasc Surg. 2009; 137:1388-93.

26. Lamade W, Vetter M, Hassenpflug P, Thorn M, Meinzer HP, Herfarth C. Navigation and image-guided HBP surgery: a review and preview. J Hepatobiliary Pancreat Surg. 2002;9:592-9.

27. Troulis MJ, Everett P, Seldin EB, Kikinis R, Kaban LB. Development of a threedimensional treatment planning system based on computed tomographic data. In J Oral Maxillofac Surg. 2002;31:349-57.

28. Fukuhara K, Akashi A, Nakane S, Tomita E. Preoperative assessment of the pulmonary artery by three-dimensional computed tomography before videoassisted thoracic surgery lobectomy. Eur J Cardiothorac Surg. 2008;34: $875-7$.

Key Words: lung cancer, posterior approach, pulmonary resection, segmentectomy, thoracoscopic 